\title{
Diagnóstico institucional: ¿cómo se construye el género en la Facultad de Teología y Humanidades de la Universidad Luterana Salvadoreña?
}

\author{
Institutional Diagnosis: How does the Theology and Humanities faculty \\ at Universidad Luterana Salvadoreña (Salvadoran Lutheran University) \\ construct gender matters?
}

\section{ISSN 2071-8748 \\ E-ISSN 2218-3345 \\ (c) (1) (2) \\ BY NC SA}

David Quintana ${ }^{2}$ ORCID: 0000-0002-6690-2360 davidquintana@uls.edu.sv

Recibido: 18/07/18 Aprobado: 12/12/18

URI: http://hdl.handle.net/11298/871

DOI: http://dx.doi.org/10.5377/entorno.v0i66.6723

\section{Resumen}

La Universidad no solo hace Docencia, Investigación y Proyección Social, voluntaria o involuntariamente también reproduce un modo de vida. Bajo esta premisa, se desarrolló un estudio cualitativo, el cual tuvo como propósito diagnosticar la construcción social de género al interior de la Facultad de Teología y Humanidades, de la Universidad Luterana Salvadoreña (ULS), a partir de las relaciones que se producen en la Comunidad Universitaria, como resultado del desarrollo del proceso enseñanzaaprendizaje, en lo concerniente al periodo 2016-2017.

En correspondencia con el objeto de estudio y objetivos del mismo, la investigación fue realizada bajo el método cualitativo, el cual propició el uso de técnicas como la observación no participante, entrevista enfocada

\section{Abstract}

The university does not only teach, research and has social outreach, voluntarily or not, but also replicates a life style. On this basis, a qualitative study was undertaken, focused on diagnosing the gender social construct at the Universidad Luterana Salvadoreña (Salvadoran Lutheran University), steaming from the relationships that occur at the university community, as a result of the development of the teaching-learning process concerning the 2016-2017 period. In correspondence with the subject of the study and its objectives, the research was conducted under the qualitative method, which favored the use of techniques such as nonparticipant observation, focused groups and focus groups. It is worth mentioning that, in accordance with

1 Este artículo surge como resultado de la elaboración de una tesis de maestría de la Universidad de El Salvador, la cual fue denominada "Relaciones dinámicas entre la Comunidad Educativa en la Construcción Social de Género" a cargo de David Quintana y Cristina Pérez.

2 Miembro de la Unidad de Investigación de la Universidad Luterana Salvadoreña. Maestro en Métodos y Técnicas de Investigación Social. Profesor de Metodología y Estadística. Email: davidquintana@uls.edu.sv 
y grupos focales. Cabe mencionar que acorde al propósito del estudio se trabajó con una muestra intencional o de conveniencia de 27 informantes claves, siendo estos Decanatos, Coordinadores de carrera, docentes y estudiantes universitarios, los cuales fueron seleccionados a partir de su accesibilidad e idoneidad de participación.

El estudio concluye, que las relaciones que se producen entre los diferentes miembros de la comunidad universitaria, resultan mediadas por una estructura jerárquica propia del sistema patriarcal. Sin embargo, es posible identificar apertura y esfuerzos desde estudiantes, docentes y coordinaciones de carrera en la construcción de relaciones más equitativas e igualitarias entre mujeres y hombres, en las que la influencia del currículo oficial y oculto pasa casi totalmente desapercibidos.

\section{Palabras clave}

Educación superior; Género; Diseño - Enseñanza; Desarrollo educativo; Trabajo social - Investigaciones. the purpose of the study, a purposive sampling of 27 key informants was used, these being deanship, career coordinators, professors and University students that were selected based on their participation suitability and accessibility. In this research, it can be concluded that the relations that take place between the different members of the University community are mediated by a hierarchical structure which is characteristic of the patriarchal system.

However, it is possible to identify the openness and efforts from students, professors and career coordinators in the construction of more equitable and equal

relationships between women and men, in which the influence of the official and hidden curriculum goes almost unnoticed.

\section{Keywords}

Higher education; Gender; Design - Teaching; Educative development; Social work - Investigations.

\section{Introducción}

En reiteradas ocasiones, el análisis de la construcción social de género en el sistema educativo salvadoreño ha sido un tema muy poco abordado desde una perspectiva crítica, a pesar de que, según Gomariz (1992), "el sistema educativo nacional, como en la mayoría de países centroamericanos, ha tenido grandes desafíos en materia de género, lo cual vuelve relevante la labor docente dentro de los centros educativos", ya que tanto mediante sus enseñanzas como de los valores que transmite influye en el alumnado con respecto a la formación de su identidad de género y, por consiguiente, en las relaciones que se establecen entre estudiante-estudiante, docente-estudiante, estudianteautoridades universitarias y con los demás miembros de la comunidad universitaria.

La educación salvadoreña, según el Ministerio de Educación (Mined, 2007), muestra, desde sus orígenes, una marcada

desigualdad de acceso. Evidencia de esta situación es que en 1957 El Salvador contaba con un sistema educativo conformado por primero y segundo ciclo de primaria y plan básico, en donde el criterio para asignar las opciones educativas era el sexo del estudiantado, lo cual generaba que a los hombres les era impartida una formación técnica tradicional, para su incorporación laboral como mano de obra productiva.

En cambio, para las mujeres el aprendizaje estaba orientado a la formación doméstica: corte y confección, bordado, cocina, pastelería y otros. Las mujeres de estratos altos tenían posibilidades educativas orientadas a la "economía doméstica", que les proporcionaba nociones de cómo administrar los bienes económicos del hogar, situación que visibilizaba la inequidad propia de la época (Mined, 2007).

"Para 1967, el Sistema Educativo Nacional estaba ya reformado con nuevos programas de estudios para 
primaria y algunos cambios para el plan básico" (Mined, 2007). Por ejemplo, con séptimo, octavo o noveno grado se podía optar por la escuela vocacional en áreas como corte y confección, "cultor" de belleza y arte y decoración. Con séptimo u octavo se preparaban taquimecanógrafas; con séptimo, octavo, noveno o décimo, se optaba para el área de Teneduría de Libros y Secretariado, siendo todavía muy poco el avance en materia de género.
Durante los períodos educativos descritos, la separación de los estudios para hombres y mujeres fue evidente. La formación técnica de los bachilleratos diversificados fortalecía una división genérica de las opciones. Por ejemplo, en el secretariado la matrícula era exclusiva para las mujeres, mientras que en la opción de automotores el predominio de los hombres era evidente. Esta situación, se muestra con datos más recientes en la tabla 1, a continuación.

Tabla 1. Matrícula en educación media, según sexo para el período 2008-2012

\begin{tabular}{|l|c|c|}
\hline Opción de bachillerato & $\begin{array}{c}\text { Mujeres } \\
\%\end{array}$ & $\begin{array}{c}\text { Hombres } \\
\%\end{array}$ \\
\hline Mecánica Industrial & 3,48 & 96,52 \\
\hline Secretariado & 96 & 4 \\
\hline Mantenimiento Automotriz & 1,35 & 98,65 \\
\hline Mecánica General & 3,28 & 96,72 \\
\hline Mecánica Naval & 21,54 & 78,46 \\
\hline Salud & 75,24 & 24,76 \\
\hline Mecánica Automotriz & 1,12 & 98,88 \\
\hline Sistemas informáticos & 18,77 & 81,23 \\
\hline Sistemas eléctricos & 3,67 & 96,33 \\
\hline Electrotecnia & 3,04 & 96,96 \\
\hline Aeronáutico & 0,36 & 99,64 \\
\hline Arquitectura & 32 & 68 \\
\hline
\end{tabular}

Fuente: elaboración propia según Mined 2008-2012.

Acorde con lo expuesto en la tabla 1, en el período 2008-2012 los porcentajes de mujeres matriculadas en especialidades que son estereotipadas como "femeninas", siendo este el caso de los bachilleratos de salud y secretariado, resultaron ser mayores en relación a la matrícula de hombres. Por otra parte, en las especialidades que tienen el prejuicio de ser "masculinas" puede observarse el mayor porcentaje de matrícula de hombres con respecto a las mujeres, siendo evidente la segmentación y las brechas de género en determinadas especialidades de educación media.
Sin embargo, desde la perspectiva del Mined (2007), "la discriminación por razón de sexo en la estructura educativa ha empezado a disminuir" en la medida que han sido abiertos los primeros espacios para discutir la aplicación del enfoque de género en la educación. Sin embargo, aún no se cuenta con los estudios que argumenten que las desigualdades en materia de género están disminuyendo al interior de las instituciones educativas, siendo este el caso de las universidades del país (ver tabla 2). 
Tabla 2. Matrícula de estudiantes universitarios, período 2010-2015

\begin{tabular}{|c|c|c|c|c|c|c|c|c|c|c|c|c|}
\hline \multirow{2}{*}{ Área } & \multicolumn{2}{|c|}{2010} & \multicolumn{2}{|c|}{2011} & \multicolumn{2}{|c|}{2012} & \multicolumn{2}{|c|}{2013} & \multicolumn{2}{|c|}{2014} & \multicolumn{2}{|c|}{2015} \\
\hline & M & $F$ & M & $F$ & M & $F$ & M & $F$ & M & $F$ & M & $F$ \\
\hline Arte y arquitectura & 2.955 & 2.519 & 3.486 & 2.954 & 3.737 & 3.134 & 3.931 & 3.418 & 3.979 & 3.759 & $s / d$ & $s / d$ \\
\hline $\begin{array}{l}\text { Economía, Administración } \\
\text { y Comercio }\end{array}$ & 15.883 & 22.628 & 17.085 & 24.110 & 18.202 & 25.030 & 18.672 & 25.664 & 18.921 & 25,824 & $s / d$ & $s / d$ \\
\hline Salud & 6.812 & 18.526 & 7.776 & 20.218 & 8.602 & 21.849 & 9.375 & 23.682 & 9.623 & 24.276 & $s / d$ & $s / d$ \\
\hline Ciencias & 1.185 & 1.525 & 1.289 & 1.535 & 1.379 & 1.690 & 1450 & 1.759 & 1.481 & 1.742 & $s / d$ & $s / d$ \\
\hline $\begin{array}{l}\text { Agropecuaria y Medio } \\
\text { Ambiente }\end{array}$ & 1.536 & 777 & 1.718 & 825 & 1.925 & 896 & 2.103 & 999 & 2.239 & 1.126 & $s / d$ & s/d \\
\hline Derecho & 6.832 & 8.815 & 6.873 & 8.759 & 6.955 & 8.713 & 7.115 & 8.909 & 6.888 & 8.830 & $s / d$ & $\mathrm{~s} / \mathrm{d}$ \\
\hline Humanidades & 2.964 & 3,649 & 3,341 & 4,535 & 3,835 & 5,127 & 4,218 & 5,771 & 4,382 & 6.149 & $s / d$ & s/d \\
\hline Tecnología & 24.020 & 8.107 & 25.222 & 8.067 & 26.447 & 8.032 & 27.003 & 7.837 & 26.406 & 7.493 & $s / d$ & s/d \\
\hline Educación & 4.199 & 10.420 & 4.777 & 10.458 & 5.306 & 11.041 & 5.031 & 10.672 & 4.752 & 9.589 & $s / d$ & s/d \\
\hline Ciencias Sociales & 2.268 & 4.302 & 2.819 & 4.557 & 2.988 & 4.972 & 3.227 & 5.227 & 3.355 & 5.479 & s/d & s/d \\
\hline Subtotal por sexo & 68.744 & 81.268 & 74.386 & 85.988 & 79.376 & 90,484 & 82.125 & 93.938 & 82.026 & 94.267 & $s / d$ & $s / d$ \\
\hline Total general & \multicolumn{2}{|c|}{150.012} & \multicolumn{2}{|c|}{160.374} & \multicolumn{2}{|c|}{169.860} & \multicolumn{2}{|c|}{176.063} & \multicolumn{2}{|c|}{176.293} & \multicolumn{2}{|c|}{$s / d$} \\
\hline
\end{tabular}

Fuente: elaboración propia, según datos de la Dirección Nacional de Educación Superior del Mined.

Como puede observarse, a pesar de que la cantidad de estudiantes universitarios ha crecido y de que el número de mujeres que están estudiando en las universidades es mayor en relación con los hombres, en carreras estereotipadas para mujeres la participación de estas continúa al alza, siendo el caso de carreras humanísticas como Educación y Ciencias Sociales.

En esta línea, Corleto (2006) sostiene que "Ios esfuerzos que a la fecha ha realizado el Ministerio de Educación de El Salvador por incorporar las perspectiva y enfoque de género en el sistema educativo solo han priorizado el marco jurídico", que comprende los tratados internacionales ratificados, las leyes nacionales y las políticas públicas, entre las que destaca la Declaración sobre la Eliminación de la Discriminación contra la Mujer" del año 2000.

Bajo esta perspectiva, el presente estudio tuvo como propósito indagar en una institución de educación superior, la ULS, de qué manera se configura la construcción social de género a partir de las relaciones dinámicas que se establecen entre los miembros de la comunidad universitaria, previa consideración de que "la Universidad Luterana Salvadoreña surgió a finales de la década de los años 80 del siglo pasado, en el contexto de un profundo conflicto social salvadoreño que propició la iniciativa de un grupo de iglesias históricas, quiénes se propusieron crear un proyecto alternativo de educación superior", que brindara oportunidades de estudios superiores a sectores empobrecidos y excluidos. (Autoestudio ULS 2014-2016).

Con esa visión, y definida la ULS como un proyecto alternativo de educación superior, esta institución ha venido trasformándose tanto a nivel interno como externo. Esto se fundamenta al considerar que "Ia ULS fue fundada el 26 de septiembre del 1987, recibiendo la personería jurídica el 23 de mayo de 1989, e iniciando sus labores académico-administrativas en febrero de 1991" (Autoestudio ULS, 2014-2016).

Técnicamente, "desde su fundación la Universidad Luterana Salvadoreña se ha caracterizado por su compromiso con la defensa y promoción de los derechos fundamentales de todo hombre y mujer, así como también por su empeño por la identificación de un nuevo proyecto de sociedad más justo, equitativo, un modelo en el que el centro de atención es el ser humano" (Autoestudio ULS 2014-2016). 
En lo referido a su filosofía educativa, acorde con el Autoestudio antes citado, "el modelo educativo de la Universidad Luterana Salvadoreña es participativo, humanista, dialógico y democrático", es decir, que está dirigido a desarrollar en los estudiantes una conciencia libre y crítica, conjugando dialécticamente la docencia, la investigación y la proyección social, vinculando el aula con la realidad nacional, en un encuentro dinámico, intenso y enriquecedor de la vida universitaria.

Desde su creación y hasta finales del año 2005, la universidad contó con subsidio de la Federación Luterana Mundial. Se estima que aproximadamente el $70 \%$ de los fondos para cubrir su presupuesto provenía de esta, y el $30 \%$ restante de las cuotas estudiantiles.

Por diversas circunstancias de índole político-ideológica, este modelo entró en crisis, sumándosele el retiro del apoyo de la Federación. Esta decisión obligó a las autoridades universitarias a diseñar una hoja de ruta que permitiera sacar adelante a la institución y continuar con el modelo alternativo de educación superior que ofrece en la actualidad.

En el año 2006, debido a la renuncia del presidente de la Junta de Directores, quien también fungía como rector y representante legal de la universidad (doctor y obispo Medardo Ernesto Gómez Soto -representante del Sínodo Luterano Salvadoreño-), se dio un cambio en dicha Junta, separándose está completamente del Sínodo, quedando integrada por profesionales independientes con experiencia en educación superior.

En agosto de 2006, la ULS puso en marcha un plan de acción en busca de su sostenibilidad, con énfasis en la obtención de recursos propios y en la construcción de una nueva cooperación nacional e internacional que permitiera la aprobación de proyectos en apoyo a la actividad académica. El plan dio buenos resultados, logrando incrementar la población estudiantil, la cual se ha quintuplicado desde entonces.

La universidad se ha caracterizado, en términos de acceso a la educación, por ser inclusiva, dado que la gran mayoría de su población estudiantil es de escasos recursos. Por ello, ha suscrito diversos convenios con instituciones nacionales e internacionales, entes gubernamentales, municipales y organizaciones no gubernamentales para poder brindar cuotas diferenciadas a los estudiantes. En ese sentido, casi el $90 \%$ de los estudiantes inscritos han sido favorecidos con los distintos convenios suscritos.

En materia de género, la ULS, a sus 26 años de historia, no cuenta físicamente con una unidad de género, a pesar de que desde el año 2015 se contrató a una profesional en calidad de coordinadora de la unidad de género que se aspira tener. En este sentido, como parte de este esfuerzo institucional, ha sido elaborada la Política de Equidad de Género, la cual se encuentra en fase de aprobación por las autoridades universitarias.

En lo referido al claustro de profesores, cuenta con "26 docentes a tiempo completo, 2 a tiempo parcial y 115 docentes contratados por hora-clase" (Autoestudio Institucional 2014-2016). En términos de matrícula, la población estudiantil asciende a un total de 2.431 estudiantes, de los cuales 580 se encuentran inscritos en el Centro Universitario Regional (institución anexa a la sede central). Al respecto, la tabla 3 muestra el crecimiento de la población estudiantil en los últimos 5 años.

Tabla 3. Población estudiantil ULS, según matrícula en el período 2011-2016

\begin{tabular}{|c|c|c|}
\hline Año & Número de estudiantes & Crecimiento interanual (\%) \\
\hline 2011 & 1.464 & $-\cdots----$ \\
\hline 2012 & 1.863 & 21,41 \\
\hline 2013 & 1.995 & 4,49 \\
\hline 2014 & 2.089 & 4,48 \\
\hline 2015 & 2.187 & 4,69 \\
\hline 2016 (ciclo 1-2016) & 2.431 & 10,03 \\
\hline
\end{tabular}

Fuente: autoestudio ULS 2014-2016. 
Hasta el año 2016, la ULS sirvió 8 carreras atendidas por dos facultades, contando cada una con su propio plan de estudios debidamente autorizado por el Mined. En estos planes están contenidos los perfiles de egreso como producto del proceso de formación de futuros profesionales, dicha responsabilidad descansa en la Facultad de Teología y Humanidades, que administra las licenciaturas en Ciencias de la Educación con especialidad en Educación Parvularia, en Trabajo Social y en Teología (carreras que participaron en esta investigación); y en la Facultad de Ciencias del Hombre y la Naturaleza, que vela por el desempeño de las carreras de Ingeniería Agroecológica y las licenciaturas en Administración de Empresas, Contaduría Pública, Ciencias de la Computación y Ciencias Jurídicas.

Asimismo, es necesario señalar que la universidad se encuentra ubicada en la Intersección Nororiente, carretera a Los Planes de Renderos, km 3 y Autopista a Comalapa, barrio San Jacinto, departamento de San Salvador.

“El campus universitario de la ULS [de la sede central] tiene una extensión de $104.414 .29 \mathrm{~m}^{2}$ (...) que albergan las oficinas administrativas y de registro académico, así como las aulas de clases" ("Catálogo Institucional ULS", 2016).

La universidad constituye un importante escenario abierto a toda su comunidad, muestra de ello es que en septiembre de 2016 fue declarada por la Organización Panamericana de Mercadeo Social como una zona 'cero discriminación y estigma'. La placa obtenida constituye un símbolo del reconocimiento y compromiso institucional adquirido, la cual se muestra en la figura 1.

\section{Figura 1. Placa zona cero discriminación}

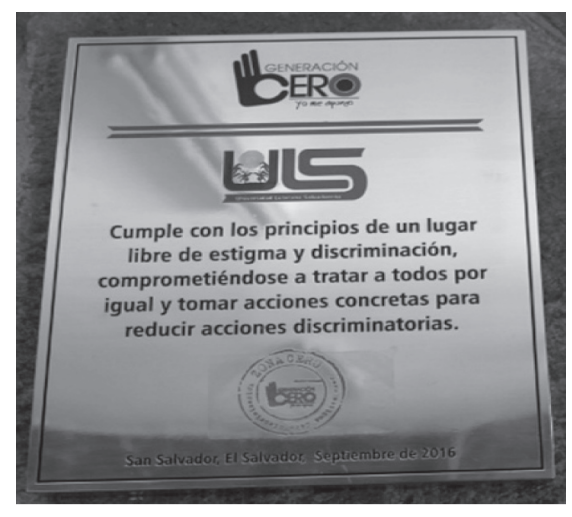

Fuente: elaboración propia

(fotografía tomada el 10 de enero 2017).
Conforme a esta caracterización del objeto de estudio, en términos operativos, la investigación fue realizada conforme los siguientes objetivos.

\section{Objetivo general}

Desarrollar una investigación que permitiera comprender la configuración de la construcción social de género en la Facultad de Teología y Humanidades de la ULS, a partir de las relaciones dinámicas que se producen en la comunidad universitaria en el desarrollo del proceso enseñanzaaprendizaje. En consecuencia de lo anterior, resultó necesario plantearse los tres objetivos específicos que se enuncian a continuación.

1. Interpretar la práctica docente a fin de determinar la influencia del currículo en la construcción de relaciones de poder mediante la organización del proceso de enseñanza-aprendizaje.

2. Describir la influencia del currículo oculto de género en la orientación del proceso de enseñanza.

3. Analizar, en la relación estudiante-estudiante, la construcción y reproducción de papeles tradicionales de género.

\section{Metodología}

Previo análisis de los objetivos del estudio, se diseñó una investigación fundamentada en el paradigma de la indagación constructivista, también denominado naturalista, hermenéutico, humanista o interpretativo, el cual, según Valles (2003), "está basado en el conocimiento que nos ayuda a mantener la vida cultural, nuestra comunicación y significados simbólicos", los cuales en este caso permitieron la comprensión de todos aquellos elementos implícitos en las relaciones dinámicas de la comunidad universitaria, que configuran la construcción social de género. En tal sentido, el desarrollo de la investigación se sustentó en la método inductivo-hipotético o cualitativo, a fin de "descubrir y comprender el significado que las personas atribuyen a su cotidianidad" desde una lógica que partió de las experiencias particulares del estudiantado, los docentes, coordinadores de carrera y las autoridades universitarias de la Facultad de Teología y Humanidades (Olabuénaga, 1989).

La investigación realizada consideró que "el objeto de estudio habla, actúa, interpreta la realidad en la cual vive" 
(Merlino, 2009). Es decir, que las experiencias solo se pudieron obtener mediante la interacción con estos sujetos, (docentes, estudiantes y autoridades universitarias), los cuales, inmersos en un contexto económico, político, social y cultural, configuraron las experiencias de aquellos.

El estudio estuvo fundamentado en el interaccionismo simbólico, según el cual el significado de una conducta se forma en la interacción social; su resultado es un sistema de significados intersubjetivos, un conjunto de símbolos de cuyo significado participan los actores, en este caso la comunidad universitaria.

El desarrollo de este estudio demandó el uso de tres técnicas, siendo estas la observación no participante, entrevista enfocada y el grupo focal. La primera de ellas, resultó útil en la medida que permitió contemplar sistemática y detenidamente tal como se desarrolla la vida cotidiana o vida social, sin manipularla, por sí mima, a la vez que permitió una interacción entre el investigador, y el fenómeno observado. (Olabuénaga: 2003).

En este sentido, se empleó la técnica de la observación de forma estructurada no participante, siendo necesario haber observado tanto dentro como fuera de aulas de clase (por ejemplo cafeterías y biblioteca) la interacción entre docentes, estudiantes y demás miembros de la comunidad universitaria, con el interés de obtener información que permitiera analizar la construcción social de género, con especial énfasis en la influencia del currículo oficial y del oculto en el desarrollo del proceso de enseñanza-aprendizaje.
La tercera de las técnicas fue "La entrevista enfocada que permite obtener información mediante una conversación profesional" (Olabuénaga: 2003). Para efectos de la presente investigación, colaboraron 2 especialistas en el tema de género, 3 docentes, 3 coordinadores/as de carrera y la decana de la Facultad de Teología y Humanidades, quienes permitieron conocer las experiencias de los diferentes actores de la comunidad universitaria, siendo fundamental para interpretar la forma en que el marco normativo institucional repercute en la construcción de relaciones de poder en los ámbitos pedagógico, organizativo y administrativo.

Finalmente, la técnica del grupo focal fue de suma importancia en la medida que "pone el acento en la creatividad y la generación de nuevas ideas a partir de un tema en discusión orientadas a un grupo de personas" (Valles, 2003), por lo que se realizaron tres grupos focales, uno por cada carrera (Educación, Trabajo Social y Teología), con 6 estudiantes ( 3 hombres y 3 mujeres previamente seleccionados), siendo favorable dado que propició una atmósfera de seguridad a los participantes, quienes no se sintieron presionados a responder cada una de las preguntas formuladas, pudiendo de este modo expresarse de manera espontánea. Cabe señalar que estos grupos focales fueron realizados en calidad de muestreo opinático.

\section{Diseño muestral}

Para Bernal (2006), la población consiste en el número total de los casos en concreto que están inmersos en un problema específico. En este estudio, la muestra total fuese de 27 participantes, como se indica en la tabla 4.

\section{Tabla 4. Determinación del muestreo teórico y opinático}

\begin{tabular}{|c|c|c|c|c|c|c|c|c|c|}
\hline $\begin{array}{c}\text { Muestreo teórico } \\
\text { (Especialistas) }\end{array}$ & \multicolumn{2}{c|}{$\begin{array}{c}\text { Muestreo opinático } \\
\text { (Decanato) }\end{array}$} & \multicolumn{2}{c|}{$\begin{array}{c}\text { Muestreo opinático } \\
\text { (Coordinadores) }\end{array}$} & \multicolumn{2}{c|}{$\begin{array}{c}\text { Muestreo opinático } \\
\text { (Profesores) }\end{array}$} & \multicolumn{2}{c|}{$\begin{array}{c}\text { Muestreo opinático } \\
\text { (Estudiantes) }\end{array}$} \\
\hline Hombres & Mujeres & Hombres & Mujeres & Hombres & Mujeres & Hombres & Mujeres & Hombres & Mujeres \\
\hline 0 & 2 & 0 & 1 & 1 & 2 & 2 & 1 & 9 & 9 \\
\hline & & & & & & & & $\begin{array}{c}(3 \text { por cada } \\
\text { carrera) }\end{array}$ & $\begin{array}{c}(3 \text { por cada } \\
\text { carrera) }\end{array}$ \\
\hline
\end{tabular}

Fuente: elaboración propia. 
Como se puede observar, el muestreo intencional permitió la participación de 12 hombres y 15 mujeres, consolidando una muestra de 27 personas.

En lo concerniente al procesamiento y análisis de los datos obtenidos en la fase de campo, se consideró que la naturaleza de los estudios cualitativos enfoca una realidad que posee una complejidad (muchas veces indecible o no consciente para el mismo sujeto hablante, tales como sus actitudes, construcciones, motivaciones, deseos y creencias).

Bajo esta perspectiva, la etapa de procesamiento inició con la codificación del lenguaje mediante una codificación inductiva, permitiendo así haberse sumergido previamente en el texto para determinar los elementos y dimensiones más relevantes, siguiendo el proceso lógico cualitativo, codificando el texto narrativo con colores (rojo, amarillo, verde, morado, naranja y azul) para cada una de las siguientes categorías teóricas establecidas: Construcción de género, Relaciones de poder, Dinámica del proceso-enseñanza, Currículo oficial y Currículo oculto, para así posteriormente haber pasado a la extracción de subcategorías.

Luego, se procedió a determinar un control de elementos espurios a fin de garantizar la fiabilidad, la veracidad de empatía y de jerarquización de sentido para realizar el oportuno control de calidad de la información. Según Olabuénaga (2003), el equipo investigador estableció los siguientes acuerdos.

1. Buscar en todo momento la proximidad con cada uno de los informantes clave en las diversas etapas del proceso de investigación.

2. Cuidar de no alterar la información vertida por los participantes, minimizando la aparición de prejuicios personales que repercutan en la interpretación de los hallazgos.

3. Considerar informantes clave a los estudiantes que cursen el $5 .^{\circ}$ año de licenciatura, coordinadores/as de las carreras determinadas, docentes hora-clase con más de 3 años de laborar en la institución.

4. Localización y ubicación de los respectivos informantes clave en su contexto (docentes, estudiantes, autoridades universitarias).
5. Contar con documentación teórica de aspectos generales de cada una de las carreras participantes en la investigación. En este sentido, resultó necesario tener un conocimiento teórico sobre el tema en particular, a fin de orientar la concreción de los significados.

6. Finalmente, se solicitó la revisión de los hallazgos y hechos formulados, con el propósito de evitar interpretaciones sesgadas (mediante la participación de dos especialistas en el tema de género).

Para efectos de validación, se priorizó la coherencia de los hallazgos logrados mediante el cruce y triangulación de la información obtenida de los diferentes informantes clave, dado que, según Valles (2003), por medio de la triangulación de datos se facilita la comprensión del fenómeno en estudio a partir de la identificación de sus medidas, tanto mediatas como inmediatas que genera la problemática.

Finalmente, se describe la aplicación de los criterios utilizados para la validación y confiabilidad de la información obtenida.

Según Valles (2003), "la credibilidad consiste en relacionar el uso de los recursos técnicos que se utilizaron para la realización de una investigación". De esta manera, se estableció el criterio de validez interna, es decir, que el equipo investigador debió sistematizar los procesos de recogida de datos, tener buen manejo de la información obtenida, de los instrumentos aplicados y de las matrices de triangulación, entre otros, los cuales permitieron realizar el estudio.

Con el objetivo de garantizar el rigor metodológico con que se planificó el estudio, el criterio de transferibilidad o validez externa, según Guba y Lincoln (1985), planteó la posibilidad de extender sus resultados a otras poblaciones. Es decir, que, tomando en cuenta que el contexto y las características de los informantes resultan similares en ambas facultades, los hallazgos de la investigación podrían transferirse o tener validez también en el contexto de la Facultad de Ciencias del Hombre y la Naturaleza de la ULS.

En esta línea, se aplicaron dos tipos de triangulación (de datos y de técnicas) para evitar los sesgos que se pueden generar por las subjetividades de los investigadores. De esta forma se estableció proximidad científica, a fin de propiciar los mínimos estándares de calidad en el procesamiento y análisis de los datos (Patton, 2002). 
Con este objetivo se realizó primeramente la triangulación de datos consistente en la verificación y comparación de la información obtenida en diferentes momentos por los actores involucrados (principalmente docentes, estudiantes y autoridades universitarias). Asimismo, la triangulación de técnicas hizo referencia al análisis de lo analizado mediante la guía de observación, las grabaciones de la guía de entrevista en profundidad y los resultados que se obtuvieron del trabajo realizado con los grupos focales. De esta manera, el equipo investigador dio solidez y confiabilidad en cuanto a la información sistematizada en el informe final.

Para la interpretación de los datos, como recurso auxiliar se consultaron fuentes bibliográficas que ayudaran a explicar, comprender y orientar la manera en que se configura la construcción social de género mediante las diversas relaciones dinámicas al interior de la comunidad universitaria, lo que facilitó el análisis de los resultados.

\section{Resultados}

Conforme a la metodología y objetivos planteados, a continuación se presentan los resultados obtenidos.

\subsubsection{Percepciones sobre género: división histórico-bio- lógica-cultural}

En las relaciones humanas, el género figura como una categoría histórico-biológica y cultural, demandando asimismo un abordaje holístico que permita su comprensión, siendo esto posible mediante el análisis de las dinámicas que se establecen de manera cotidiana en las relaciones de los seres humanos en su contexto, siendo este el caso de las interacciones que se producen en el desarrollo del proceso enseñanza-aprendizaje, donde, para efectos de la presente investigación, converge la comunidad educativa de la ULS.

Como lo expresa la decana de la Facultad de Teología y Humanidades, "el género se identifica a través del sexo femenino y masculino; es una división de hombres y mujeres" (entrevista personal, 9 de mayo de 2017). En esta línea, fue posible identificar confusiones entre los términos sexo y género, regularmente utilizados como sinónimos de manera errónea.

Esta concepción de género, se amplía con la opinión de los coordinadores de carrera, como se muestra en la tabla 5.

Tabla 5. Género, según coordinadores/as de carrera

\begin{tabular}{|c|c|c|}
\hline $\begin{array}{c}\text { Coordinadora } \\
\text { Licenciatura en Educación }\end{array}$ & $\begin{array}{l}\text { Coordinadora } \\
\text { Licenciatura en Trabajo Social }\end{array}$ & $\begin{array}{c}\text { Coordinador } \\
\text { Licenciatura en Teología }\end{array}$ \\
\hline $\begin{array}{l}\text { "Es un concepto social que se ha ido } \\
\text { construyendo a partir de las funciones que } \\
\text { realizan el hombre o la mujer; y que no habría } \\
\text { limitante para poder desarrollar diferentes } \\
\text { acciones, actividades o funciones, sino más bien } \\
\text { separábamos el concepto mujer-hombre, pero } \\
\text { que en realidad ambos son capaces de realizar } \\
\text { las mismas actividades." }\end{array}$ & $\begin{array}{l}\text { "El género son las relaciones } \\
\text { interpersonales que vivimos a diario; } \\
\text { el encontrarnos, el trabajar, hombres y } \\
\text { mujeres." }\end{array}$ & $\begin{array}{l}\text { "Según tengo entendido, se divide en } \\
\text { dos: femenino y masculino. El género } \\
\text { tiene que ver, ya, con cuestiones } \\
\text { biológicas." }\end{array}$ \\
\hline
\end{tabular}

Fuente: elaboración propia a partir de los resultados de la guía de entrevista a coordinadores/as de carrera. 
Conforme lo expresado por los jefes de carrera, se tiene una multiplicidad de concepciones, puesto que, para la coordinadora de la carrera de Ciencias de la Educación, hablar de género significa hablar de construcciones que la sociedad ha creado a partir de las diferencias biológicas entre hombres y mujeres. En cambio, para la coordinadora de la carrera de Trabajo Social, el género lo constituye el diario vivir mediante las relaciones interpersonales que se estable cen entre hombres y mujeres sin distinción alguna. En esta línea, el coordinador de la carrera de
Teología coincide con la coordinadora de Educación, quien sostiene que la diferenciación de los géneros masculino y femenino obedece a una división biológica de la humanidad.

En lo que refiere al sector docente, es posible observar que concuerdan con que la categoría Género se define como una construcción social que determina los papeles para mujeres y para hombres dentro de la sociedad. Estas afirmaciones se exponen a continuación en la tabla 6.

Tabla 6. Género, desde la perspectiva docente

\begin{tabular}{|l|l|l|}
\hline \multicolumn{1}{|c|}{$\begin{array}{c}\text { Docente } \\
\text { Licenciatura en Educación }\end{array}$} & \multicolumn{1}{c|}{$\begin{array}{c}\text { Docente } \\
\text { Licenciatura en Trabajo Social }\end{array}$} & $\begin{array}{c}\text { Docente } \\
\text { Licenciatura en Teología }\end{array}$ \\
\hline $\begin{array}{l}\text { "Es desde la construcción social, la } \\
\text { perspectiva de género en primer lugar } \\
\text { lo ideal sería mantener una situación de } \\
\text { equilibrio o equidad" }\end{array}$ & $\begin{array}{l}\text { estamos hablando de género, hablamos } \\
\text { de características. En este caso algunos } \\
\text { privilegios asignados tanto a hombres como } \\
\text { a mujeres, y que marcan la diferencia entre } \\
\text { hombres y mujeres" }\end{array}$ & $\begin{array}{l}\text { que se ha dado, la cual define lo que es } \\
\text { apropiado para el sexo, tanto masculino } \\
\text { comenino." }\end{array}$ \\
\hline
\end{tabular}

Fuente: elaboración propia a partir de los resultados de la guía de entrevista a docentes.

A pesar de los continuos esfuerzos en la búsqueda de una sociedad más justa, históricamente las relaciones entre mujeres y hombres han sido marcadas por un sistema patriarcal que favorece la condición económica, política y social de los hombres como consecuencia del sometimiento y sacrificio de las mujeres. Esta situación, según entrevista realizada a Deysi Cheyne el 29 de marzo de 2017, radica en que "el machismo en nuestras sociedades se expresa en la creencia, en la convicción firme de los hombres, de que ellos son superiores a las a mujeres; (...) si yo soy superior, aquellos otros seres humanos, que son las mujeres, son inferiores; y por naturaleza tienen que tener tales comportamientos subordinados a las decisiones de los hombres".

Como consecuencia, se tiene la formación de una cultura androcentrista y machista, transmitida y aceptada en muchas ocasiones sin crítica alguna, de generación en generación, lo cual ha llegado hasta la academia.
Conforme con el primero de los objetivos de investigación, siendo este interpretar la práctica docente a fin de determinar la influencia del currículo en la construcción de relaciones de poder mediante la organización del proceso enseñanza-aprendizaje, el estudio encontró lo siguiente.

En las coordinaciones de carrera, el tema de género a nivel universitario tiene mucha apertura. No obstante, según lo expuesto por la coordinadora de la Licenciatura de Trabajo Social, si bien es cierto que se incentiva a abordar el tema de género, el docente tiene libertad de cátedra, por lo que no se puede afirmar que el cien por ciento del cuerpo docente lo esté incorporando en el currículo de su cátedra. Por su parte, el coordinador de la Licenciatura en Teología asume que en el pénsum de la carrera no se cuenta con ninguna materia donde se aborde el tema de género, pero que se está en el proceso de reformar el pénsum para incorporarlo. Estas afirmaciones se exponen en la tabla 7. 
Tabla 7. Influencia del currículo oficial en la orientación del proceso enseñanza-aprendizaje, según coordinaciones de carreras

\begin{tabular}{|l|l|l|}
\hline \multicolumn{1}{|c|}{$\begin{array}{c}\text { Coordinadora } \\
\text { Licenciatura en Educación }\end{array}$} & $\begin{array}{c}\text { Coordinadora } \\
\text { Licenciatura en Trabajo Social }\end{array}$ & $\begin{array}{c}\text { Coordinador } \\
\text { Licenciatura en Teología }\end{array}$ \\
\hline "Yo creo que, como institución, se tiene \\
$\begin{array}{l}\text { mucha apertura que favorece a estos } \\
\text { temas porque tienen un enfoque social." }\end{array}$ & $\begin{array}{l}\text { "De alguna manera incentiva al docente } \\
\text { libertad de cátedra, no podría decir que } \\
\text { el cien por ciento se está desarrollando } \\
\text { en el currículo de cada cátedra." }\end{array}$ & $\begin{array}{l}\text { "El pénsum que tenemos y todo el quehacer de } \\
\text { la carrera no tiene ninguna materia dedicada } \\
\text { reconstruir el pénsum; y tenemos que meter } \\
\text { esa materia porque es necesaria." }\end{array}$ \\
\hline
\end{tabular}

Fuente: elaboración propia a partir de los resultados de la entrevista a coordinadores de carrera.

Por otra parte, con el propósito de describir la influencia del currículo oculto de género en la orientación del proceso-enseñanza, siendo este uno de los objetivos de la investigación, se parte de la premisa que, según Hierro,

la universidad contribuye a la transmisión de pautas sobre las relaciones de género en forma explícita o implícita, a través de la presencia o ausencia de perspectiva de género en los contenidos curriculares, en las actividades de investigación, docencia o extensión y mediante el tipo de relaciones que predominan en sus pautas de funcionamiento y en su cultura organizacional (2003, p. 93).
Es decir, a partir de las directrices de un currículo oficial y uno oculto que orientan el desarrollo del quehacer universitario.

En este sentido, los docentes de las licenciaturas en Educación, Trabajo Social y Teología concuerdan con que el personal docente ejerce una influencia directa en la construcción y reproducción de género en el ámbito Universitario y que se manifiestan en la interacción con el estudiantado, situación que se expone a continuación en la tabla 8.

Tabla 8. Influencia del currículo oculto en la orientación del proceso enseñanza-aprendizaje, según docentes

\begin{tabular}{|c|c|c|c|c|c|c|c|c|c|}
\hline \multicolumn{2}{|c|}{$\begin{array}{c}\text { Muestreo Teórico } \\
\text { (Especialistas) }\end{array}$} & \multicolumn{2}{c|}{$\begin{array}{c}\text { Muestreo opinático } \\
\text { (Decanato) }\end{array}$} & \multicolumn{2}{c|}{$\begin{array}{c}\text { Muestreo opinático } \\
\text { (Coordinadores) }\end{array}$} & \multicolumn{2}{c|}{$\begin{array}{c}\text { Muestreo opinático } \\
\text { (Profesores) }\end{array}$} & \multicolumn{2}{c|}{$\begin{array}{c}\text { Muestreo opinático } \\
\text { (Estudiantes) }\end{array}$} \\
\hline Hombres & Mujeres & Hombres & Mujeres & Hombres & Mujeres & Hombres & Mujeres & Hombres & Mujeres \\
\hline 0 & 2 & 0 & 1 & 1 & 2 & 2 & 1 & 9 & 9 \\
\hline & & & & & & & & $\begin{array}{c}(3 \text { por cada } \\
\text { carrera) }\end{array}$ & $\begin{array}{c}(3 \text { por cada } \\
\text { carrera) }\end{array}$ \\
\hline
\end{tabular}

Fuente: elaboración propia a partir de los resultados de la entrevista dirigida a docentes. 
Acorde con lo expuesto por el claustro docente, queda en evidencia que reconocen teóricamente la influencia del currículo oculto en la orientación del proceso enseñanzaaprendizaje, a la vez que destacan lo positivo y negativo que puede generar en la orientación del mencionado proceso. Muestra de esta situación es que en el caso del docente de la carrera de Educación este currículo, el cual no es medido ni observado por nadie, ha sido un factor positivo en el desarrollo del proceso enseñanza-aprendizaje (PEA).

De igual manera, con el interés de analizar en la relación estudiante-estudiante la construcción y reproducción de papeles tradicionales de género a partir de las relaciones dinámicas entre la comunidad universitaria, es decir, donde interactúan docentes, estudiantes, personal administrativo y autoridades universitarias, a continuación los actores exponen sus planteamientos.
Para el coordinador de la Licenciatura en Teología "nuestra idea no es así verticalista sino que lo hacemos en cuestiones más horizontales, más de respeto mutuo más que de ejercer un poder vertical", lo cual presupone la búsqueda de relaciones armónicas con la Comunidad Universitaria en las que se minimiza el verticalismo mediante el ejercicio del poder. (Entrevista personal, 27 de marzo de 2017.)

En esta línea, los estudiantes de las tres carreras sostienen que la dinámica de sus relaciones interpersonales, al momento de realizar diversas tareas grupales en las que el trabajo en equipo demanda la necesidad de interactuar, asumen diversos papeles, sin discriminación negativa, como se expone en la tabla 9.

\section{Tabla 9. Dinámica de distribución de papeles de trabajo, según estudiantes universitarios}

\begin{tabular}{|c|c|c|}
\hline $\begin{array}{c}\text { Estudiantes } \\
\text { Licenciatura en Educación }\end{array}$ & $\begin{array}{l}\text { Estudiantes } \\
\text { Licenciatura en Trabajo Social }\end{array}$ & $\begin{array}{c}\text { Estudiantes } \\
\text { Licenciatura en Teología }\end{array}$ \\
\hline $\begin{array}{l}\text { "Bien. Nosotros, en mi grupo, fijese } \\
\text { que, nosotros, ahí vemos primero la } \\
\text { responsabilidad de cada quien. Uno se } \\
\text { conoce; y realmente vemos las características } \\
\text { que cada uno de nosotros tengamos. Así es } \\
\text { como se va a distribuir el trabajo, porque no } \\
\text { todos somos buenos en todo; así como las } \\
\text { inteligencias múltiples. Si tengo que hacer un } \\
\text { mural, veo las cualidades de cada quien; y así } \\
\text { vamos delegando las responsabilidades." }\end{array}$ & $\begin{array}{l}\text { "Yo pienso que en todos los grupos } \\
\text { siempre hay un líder que lleva la iniciativa. } \\
\text { En el caso de nosotros, a veces yo, a veces } \\
\text { él, asignamos el trabajo que creemos que } \\
\text { pueden hacer; que tienen la capacidad, } \\
\text { aunque yo sea mujer y él sea hombre, } \\
\text { los dos tenemos la misma capacidad de } \\
\text { hacer las cosas. Igual, hay cosas que yo } \\
\text { no las puedo hacer; y hay cosas que ellos } \\
\text { sí las pueden hacer; es como darle la } \\
\text { oportunidad a los dos (géneros)." }\end{array}$ & $\begin{array}{l}\text { "No es cuestión de género sino de la } \\
\text { capacidad de cada quien. Como algunos } \\
\text { tienen diferentes habilidades, por ejemplo, } \\
\text { algunos son buenos en la oratoria, entonces } \\
\text { se dice: 'yo voy a hacer el instrumento, vos } \\
\text { exponé'. Entonces no se parte en ningún } \\
\text { momento por el género." }\end{array}$ \\
\hline
\end{tabular}

Fuente: elaboración propia, a partir de los resultados del grupo focal con estudiantes de las tres carreras participantes.

Por lo aquí abordado, el establecimiento de papeles dentro de los equipos de trabajo, en opinión de los estudiantes, obedece a un análisis de las competencias que cada uno posee, es decir, a partir de su capacidad y aporte al logro de los objetivos, lo cual posibilita la participación de los estudiantes, y, por el contrario, reduce positivamente la existencia de situaciones discriminatorias entre los estudiantes, dado que estos afirman que el género no es visto como un factor que posibilite o que limite el aporte que cada uno puede dar en los procesos de aprendizaje. 


\section{Hallazgos}

El desarrollo de la investigación propició la formación de los siguientes hallazgos:

1. Los esfuerzos de sensibilización realizados por la ULS en materia de género (diplomados, reflexiones, foros, discusiones, análisis, talleres, conferencias, entre otros.) carecen de una articulación y consistencia que permita el desarrollo de un proceso sistemático y permanente.

2. En términos generales, el claustro docente ha tenido, a título individual, alguna experiencia no sistemática en el tema de género. Sin embargo, tales experiencias han sido fuera de la universidad, donde se ha desarrollado una serie de actividades de sensibilización, regularmente dirigidas hacia la población estudiantil. Al respecto, se considera que esta situación obedece a la inexistencia y a la necesidad de implantar una política institucional que aborde de manera transversal el tema de equidad e igualdad de género en el quehacer de la ULS.

3. De las tres carreras participantes en la investigación, la Licenciatura en Trabajo Social evidencia mayor compromiso institucional por el abordaje que hace del tema de género; muestra de ello lo constituye el contar en su malla curricular con cátedras donde se analiza la problemática, así como también con un amplio número de talleres, foros, conferencias, entre otros, los cuales han sido orientados en pro de la sensibilización.

4. La comunidad universitaria muestra apertura al análisis de la problemática de género, lo cual les permite visibilizar desigualdades y reproducción de papeles tradicionales en la estructura organizativa y las actividades de la ULS.

5. La ULS carece de protocolos que le permitan la detección de y atención a casos de violencia de género, así como de una articulación interinstitucional efectiva con el Mined y el Instituto Salvadoreño para el Desarrollo de la Mujer, lo cual no permite optimizar esfuerzos en materia de equidad e igualdad de género.

\section{Conclusiones finales}

En concordancia con los objetivos del estudio, finalmente fue posible formular las conclusiones que se enuncian a continuación.

1. En lo que refiere a la Facultad de Teología y Humanidades de la ULS, la labor docente se desarrolla bajo las directrices de un currículo, que carece de manera transversal del enfoque de género. Sin embargo, el hecho de contar con docentes que tienen algún tipo de sensibilización en el tema de género ha favorecido que el PEA se organice y desarrolle en un clima de relaciones de poder en las que se propicia la horizontalidad.

2. El currículo oculto de género continúa siendo un factor omnipresente en el desarrollo del proceso enseñanza-aprendizaje en la ULS, expresándose en ocasiones esporádicas mediante el empleo de chistes y vocabulario sexista en la relación docente-estudiante y estudiante-estudiante, quienes a su vez presentan dificultades para identificar sus repercusiones como mecanismo de violencia y reproducción de papeles de género, situación que favorece que su influencia pase desapercibida casi en su totalidad por la comunidad universitaria.

3. En términos generales, la relación estudiante-estudiante se desarrolla al interior del campus universitario bajo un clima de relaciones armoniosas en las que se valora de manera primordial las competencias propias de cada uno, reduciendo así las posibilidades de discriminación por razón de género.

4. Las relaciones que se producen entre los miembros de la comunidad universitaria resultan mediadas por una estructura jerárquica propia del sistema patriarcal, sin embargo, es posible identificar apertura y esfuerzos desarticulados de parte de estudiantes, docentes y coordinaciones de carrera en la construcción de relaciones más equitativas e igualitarias entre mujeres y hombres, en las que la influencia del currículo oficial y del oculto pasan casi totalmente desapercibidos.

\section{Referencias}

Bernal, C. (2006). Metodología de la Investigación. (2.a ed.). México: Pearson. Educación.

Corleto, A. (2010). "Sistema de Indicadores para la equidad de género en las Instituciones de Educación Superior". México, D.F. Universidad Nacional Autónoma de México, Programa Universitario de Estudios de Género e Instituto Nacional de las Mujeres.

Gomariz, E. (1992). "Los estudios de género y sus fuentes epistemológicas. Periodización y perspectivas", en ISIS Internacional $\mathrm{n} .^{\circ} 17$, Santiago de Chile.

Merlino. A. (2009) Investigación Cualitativa en Ciencias Sociales. (1.a ed.), Buenos Aires, Argentina. 
Mined (2007). Boletín n. ⒚ “21 de junio, día de la educación no sexista; mecanismos para la equidad de género en la educación".

Patton, M. (2002). Métodos Cualitativos de Investigación y Evaluación. (3. a ed.) Thousand oaks, California: Publicaciones SAGE.

Ruiz Olabuénaga, (1989). La decodificación de la vida cotidiana: Métodos de Investigación Cualitativa. Bilbao: Publicaciones Universidad de Deusto.

Ruiz Olabuénaga, J.I. (2003). Metodología de la Investigación Cualitativa. (3. ${ }^{a}$ ed.). España: Ediciones Aljibe.

ULS (2016) Autoestudio 2014-2016, San Salvador, El Salvador. ULS (2016). Catálogo Institucional, San Salvador, El Salvador.
Valle, M.S. (2003). Técnicas Cualitativas de Investigación Social: Reflexión Metodológica y Práctica Profesional. Madrid: Editorial Síntesis, S.A.

\section{Referencias electrónicas}

Lincoln, Y.S. \& Guba, E. (1984). Naturalistic inquiry. California: Sage Publications.

Hierro, G. (2003). “Género y educación”. Disponible en http:// www.udg. $\mathrm{mx} / \mathrm{la}$ ventana/libbr2/hierro.html

Silves, C. y Delgado, G. (2014). "Teoría de Género. ¿De qué estamos hablando? 5 claves para el debate". Disponible en http://www.ieschile.cl/claves/teoria.pdf 